\title{
Electro-Viscoelastic Migration under Simultaneously Applied Microfluidic Pressure-Driven Flow and Electric Field
}

\author{
Murat Serhatlioglu, Ziya Isiksacan, Melis Özkan, Dönüs Tuncel, and Caglar Elbuken* \\ Cite This: Anal. Chem. 2020, 92, 6932-6940 \\ Read Online
}

ABSTRACT: Under the simultaneous use of pressure-driven flow and DC electric field, migration of particles inside microfluidic channels exhibits intricate focusing dynamics. Available experimental and analytical studies fall short in giving a thorough explanation to particle equilibrium states. Also, the understanding is so far limited to the results based on Newtonian and neutral viscoelastic carrier fluids. Hence, a holistic approach is taken in this study to elaborate the interplay of governing electrophoretic and slip-induced/elastic/ shear gradient lift forces. First, we carried out experimental studies on particle migration in Newtonian, neutral viscoelastic, and polyelectrolyte viscoelastic media to provide a comprehensive understanding of particle migration. The experiments with the viscoelastic media led to contradictory results with the existing explanations. Then, we introduced the

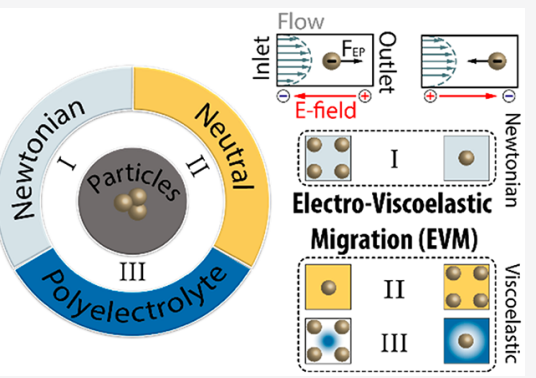
Electro-Viscoelastic Migration (EVM) theory to give a unifying explanation to particle migration in Newtonian and viscoelastic solutions. Confocal imaging with fluorescent-labeled polymer solutions was used to explore the underlying migration behavior. A surprising outcome of our results is the formation of cross-sectionally nonuniform viscoelasticity that may have unique applications in microfluidic particle focusing.

$\mathrm{M}$ icrofluidic devices enable precise control on particle migration in Newtonian and viscoelastic media under pressure-driven flow, which leads to a rich spectrum of applications, including counting, ${ }^{1-5}$ focusing, ${ }^{6-8}$ and sorting $^{9-13}$ of particles. Simultaneously applying a DC electric field with pressure-driven flow brings a new modality to tune particle equilibrium positions. The electric field induces a lateral electrophoretic force on the charged particles, thereby generating particle motion relative to the direction of the flow. However, the observations of particle migration under simultaneous pressure-driven flow and DC electric field yielded anomalous cross-sectional particle movement. ${ }^{14}$ DNA molecules migrated toward the capillary center when electrophoretic force was in the same direction as the pressure flow (concurrent) and toward the capillary wall when the pressure flow direction was reversed (countercurrent). Analytical and experimental studies have been conducted to understand the physics underlying the cross-sectional migration under simultaneous pressure-driven flow and DC electric field. We categorize these studies into four groups, as shown in Figure 1: (I) polyelectrolyte migration in Newtonian medium, (II) particle migration in Newtonian medium, (III) polyelectrolyte migration in viscoelastic medium, and (IV) particle migration in viscoelastic medium.

The principles of particle migration behavior observed in these four categories (I-IV) have been investigated in the literature. (I) Polyelectrolyte $\lambda$-DNA migrated toward the center (wall) in the concurrent (countercurrent) case in the Newtonian buffer solution. This migration was attributed to the weak electro-hydrodynamic interactions and dipolar flow fields between the DNA segments. ${ }^{15-18}$ (II) Charged particles (polystyrene beads, human cells) migrated toward the wall (center) in the concurrent (countercurrent) case in the Newtonian buffer solution. This migration was attributed to slip velocity, which is the velocity difference between the particle and the fluid. Slip velocity interacts with the local shear field and causes a net cross-stream migration. ${ }^{19-23}$ (III) Polyelectrolyte DNA samples migrated toward the center (wall) in the concurrent (countercurrent) in polyvinylpyrrolidone-based viscoelastic solutions. ${ }^{24,25}$ The amplitude and direction of the electric field modulate the shear around the DNA, which consequently affects the elastic lift force. Thus, the direction of the lift force flips according to the modulated shear. (IV) Charged polystyrene (PS) particles suspended in poly(ethylene oxide) (PEO)-based viscoelastic solution migrated toward the center (wall) in the concurrent (countercurrent) case. The results were attributed to the electrophoretic motion-induced extra lift force, originated from the nonlinear coupling of an external force-driven particle and the local viscoelastic shear flow, in the viscoelastic fluid. ${ }^{21}$ An indepth theoretical discussion of particle migration in viscoelastic flow under the external electric field is recently given. ${ }^{26}$

Received: December 12, 2019

Accepted: April 16, 2020

Published: April 16, 2020 


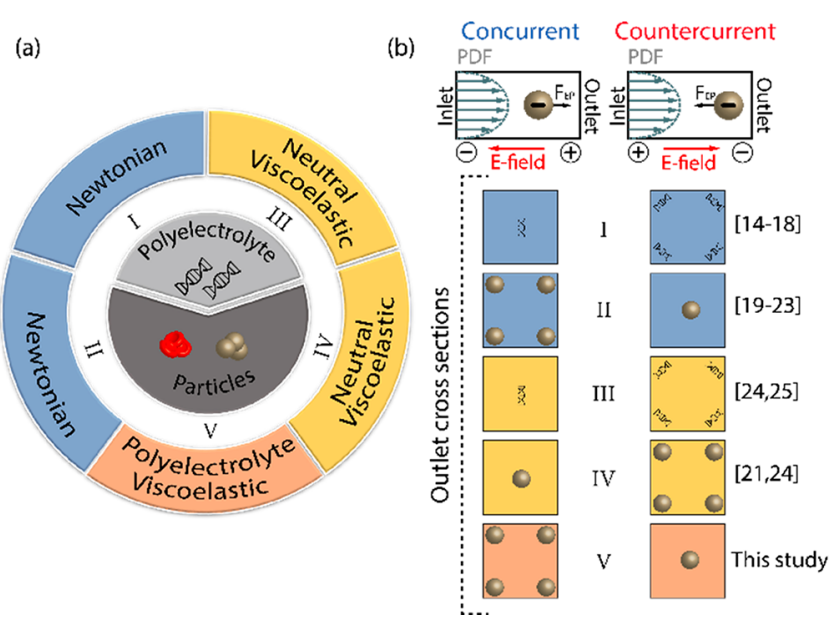

Figure 1. Schematic illustration of the reported studies in the literature for migration under simultaneously applied pressure-driven flow and electric field configuration. (a) Studies are categorized according to the medium (outer ring) and the particles of interest (inner circle) as a pie chart. (b) Schematic of the particle equilibrium states at the outlet cross sections in a microfluidic channel according to the electric field direction, medium, and suspended particles. The first four groups (I-IV) are cited from the literature, and the fifth group (V) represents our study for particle migration in polyelectrolyte viscoelastic solution. $F_{\mathrm{EP}}$ : electrophoretic force, PDF: Pressure-driven flow.

Here, we introduce a new group, the fifth group (V), particle migration in a polyelectrolyte viscoelastic medium, and introduce Electro-Viscoelastic Migration (EVM) to explain the migration behaviors in groups III, IV, and V. Consequently, we propose a unifying hypothesis to explain the underlying mechanism of cross-sectional particle migration in microchannels.

In our study, we performed particle migration experiments with PS particles suspended in (II) deionized (DI) water as well as (IV) PEO-based neutral and (V) hyaluronic acid (HA)based polyelectrolyte viscoelastic solutions in a microfluidic channel under simultaneous use of pressure-driven flow and DC electric field (Figure 2). We observed particle equilibrium positions at the microchannel, $1 \mathrm{~cm}$ away from the outlet, under an optical microscope. In DI water, particles migrated toward the channel wall in the concurrent case and toward the channel center in the countercurrent case. The same results were obtained for HA-based viscoelastic solutions, whereas particle equilibrium positions were reversed for PEO-based solutions. Although both PEO and HA have viscoelastic properties, the steady-state migration profile surprisingly exhibited opposite migration results.

We believe that the anomalous cross-stream migration difference between PEO- and HA-based solutions is due to the polyelectrolyte nature of $\mathrm{HA}^{27} \mathrm{We}$ showed that electrophoretic force affects the HA polyelectrolyte due to the charged nature of the polymer, unlike PEO neutral polymers. This generates a net cross-stream migration for both HA polymers and PS particles, which is the opposite of usual particle migration behavior in neutral viscoelastic solutions, as demonstrated by our confocal microscopy experiments. To explain this particle migration behavior in viscoelastic solutions, we propose the EVM theory.
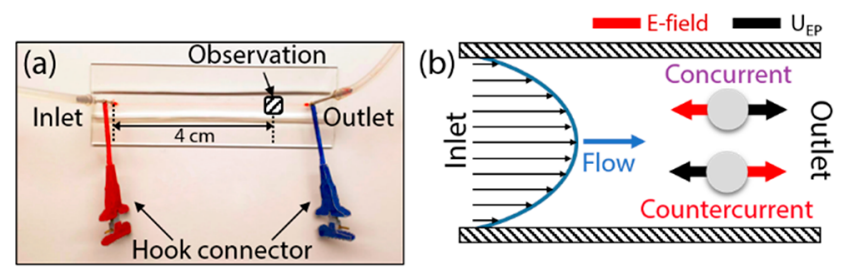

(d)
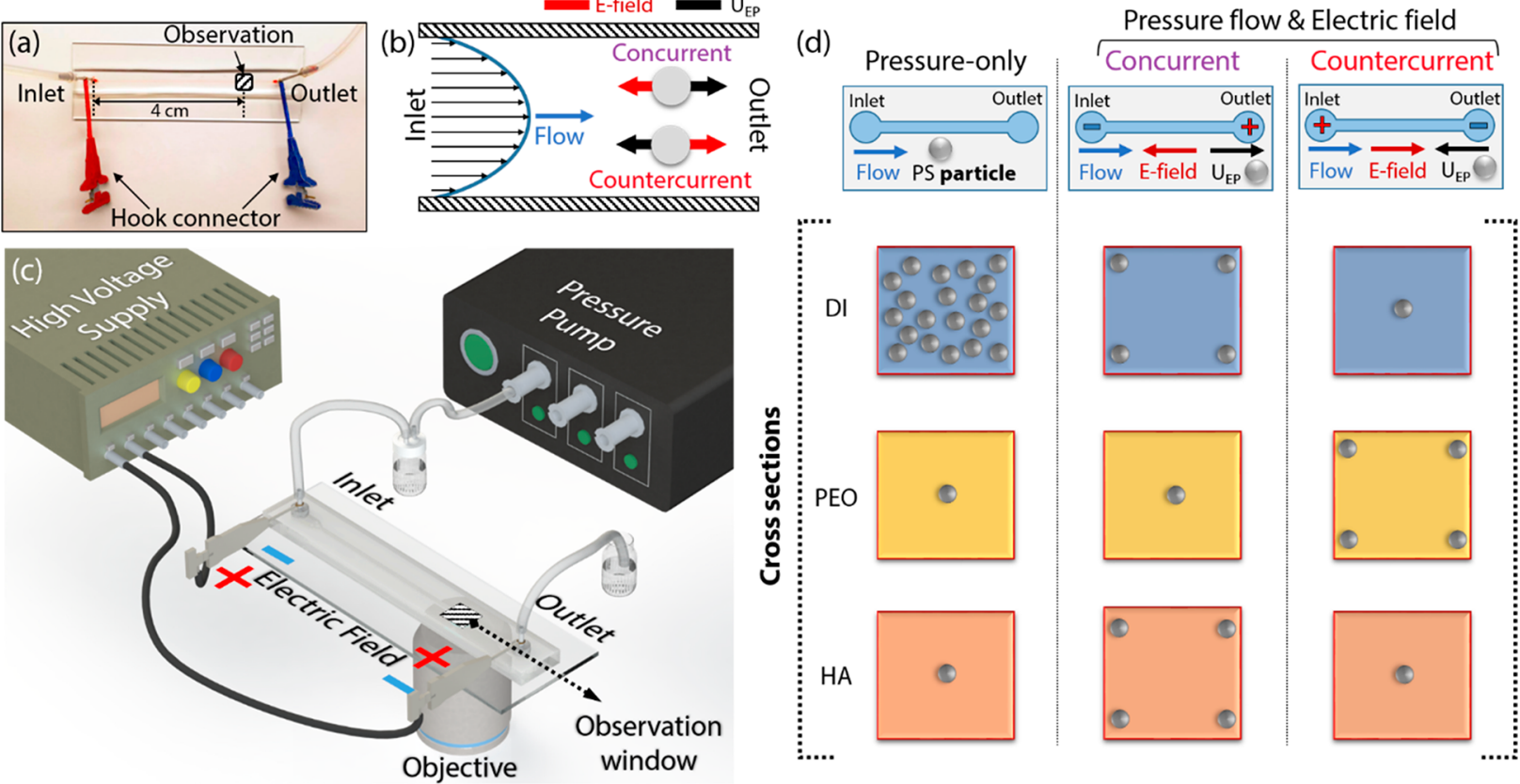

Figure 2. Illustration of the microfluidic test setup and summary of particle equilibrium states at the microchannel outlet cross sections. (a) Photo of the fabricated microfluidic chip. (b) Illustration of the electric field, electrophoretic force and Poiseuille flow distribution in the microchannel. (c) Schematic representation of the experimental system. Particle suspensions were introduced through the inlet of the channel with a pressure pump. The electric field was simultaneously applied along the channel by a high voltage supply using hook connectors. Particle migration was observed at the outlet. (d) Illustration of particle equilibrium positions at the outlet cross section for pressure-only, concurrent and countercurrent experiments. Particles in PEO- and HA-based viscoelastic solutions showed similar migration trends in pressure-only case. On the other hand, for concurrent and countercurrent cases, particle equilibrium positions were opposite for two viscoelastic solutions. Interestingly, particles in HA viscoelastic solutions showed a similar migration trend to DI water. 


\section{MATERIALS AND METHODS}

Device Fabrication. Microchannel fabrication was carried out in a Class 100 cleanroom using conventional soft lithography techniques. First, the silicon mold was fabricated. A $50 \mu \mathrm{m}$ thick SU-8 2050 photoresist (Microchem) was spincoated onto a 4 inch silicon wafer with two steps of spinning: $55 \mathrm{~s}, 500 \mathrm{rpm}$, and $40 \mathrm{~s}, 3500 \mathrm{rpm}$ followed by soft bake (2 min at $65{ }^{\circ} \mathrm{C}, 7 \mathrm{~min}$ at $95{ }^{\circ} \mathrm{C}, 1 \mathrm{~min}$ at $\left.65^{\circ} \mathrm{C}\right)$, UV exposure $\left(180 \mathrm{~mJ} / \mathrm{cm}^{2}\right)$, post bake $\left(3 \mathrm{~min}\right.$ at $65{ }^{\circ} \mathrm{C}, 7 \mathrm{~min}$ at $95^{\circ} \mathrm{C}, 1$ min at $65{ }^{\circ} \mathrm{C}$ ), and development (5 min). Then, PDMS (Dowcorning Sylgard 184) was mixed with the curing agent at a 10:1 ratio. The mixture was degassed in a vacuum chamber, poured onto the mold, and cured on a hot plate for $5 \mathrm{~h}$ at 90 ${ }^{\circ} \mathrm{C}$. Cured PDMS was peeled off from the mold, and $1 \mathrm{~mm}$ holes were punched for inlet and outlet connections. Finally, the PDMS layer (microchannel dimensions: $50 \mathrm{~mm}$ length, 60 $\mu \mathrm{m}$ width, $50 \mu \mathrm{m}$ height) was plasma-bonded to a glass slide to form the microchannel.

Experimental Setup. Gauge 20 syringe tips (Jensen Global) were used for tubing connections. Polypropylene hubs were peeled-off from the metal tips, and the remaining stainless steel cannula was connected to $10 \mathrm{~mm}$ long Tygon tubing (ID: $0.796 \mathrm{~mm}$ ), one for the inlet and another one for the outlet. High voltage DC power supply (LabSmith HVS4448-3000 V) was connected to the stainless steel metal tips using hook connectors. The sample flow was pumped from the inlet with a pressure pump (Elveflow OB1).

Particle migration in the microchannel was monitored with a high-speed camera (Phantom Miro e2) at $3000 \mathrm{fps}$ under an inverted microscope (ZEISS Axio Vert.A1). Recorded videos were split to single frames and image-stacked to form a single photo with a MATLAB script to show the trajectory of the particles.

A Leica spectral inverted SP8 lightening confocal microscope with an automatic stage control was used for confocal microscopy imaging of the fluorescent dye-labeled PEO and HA viscoelastic solutions. For confocal imaging, 488 and 405 $\mathrm{nm}$ wavelength excitation laser was used for PEO and HA, respectively. Leica HCX PL FILUOTAR L 20×/0.40 NA dry microscope objective was used for imaging. A galvo-resonant scanner was used for the imaging where the image scanning frequency was $200 \mathrm{~Hz}$ per frame, whereas the frame size or the imaging area was 512 pixels $(129.0 \mu \mathrm{m} ; x) \times 512$ pixels $(129.0$ $\mu \mathrm{m} ; y)$. Total number of 40 frames are $Z$-stacked. The slice thickness along $z$ was $1.56 \mu \mathrm{m}$, while the scanning pixel size was $0.253 \mu \mathrm{m}$ both in $x$ and $y$. The rest of the setup and experimental conditions were the same as the particle migration experiments.

Sample Preparation. Viscoelastic solutions were prepared by dissolving polymer (PEO, HA) powder in DI water at the given concentration. Three different particle suspensions were prepared for the particle migration experiments: DI water, 500 ppm 5 MDa PEO-based (Sigma-Aldrich), and 1000 ppm 1.5 MDa HA-based (NewDirections) viscoelastic solutions. A total of 10 different particle suspensions were prepared: 2000, 1000, 500,100 , and $50 \mathrm{ppm}$ of $5 \mathrm{MDa}$ PEO-based and 5000, 2000, 1000, 200, and $100 \mathrm{ppm}$ of $1.5 \mathrm{MDa}$ HA-based viscoelastic solutions. Shear-dependent viscosities of solutions were measured using a rotational rheometer (Anton Paar, MCR 301). The $6 \mu \mathrm{m}$ diameter spherical polystyrene particles (Polysciences, Inc.) were suspended with the same concentrations $\left(10^{3}\right.$ particles $\left./ \mathrm{mL}\right)$ in each solution. During the experiments, particles at the inlet vial were continuously mixed with a magnetic stirrer to keep the particle concentration uniform.

Particle zeta potentials in suspensions were measured in Malvern Nano ZS ZEN3600 zeta sizer as $-60 \mathrm{mV}$ in DI water, $-80 \mathrm{mV}$ in $\mathrm{PEO}$, and $-110 \mathrm{mV}$ in HA.

Labeling of PEO and HA with Fluorescent Dyes. The fluorescent dyes green-Coumarin 343 and blue-DAPI, N-(3(dimethylamino)propyl)- $N^{\prime}$-ethyl carbodiimide hydrochloride (EDC), and 4-(dimethylamino) pyridine (DMAP) were purchased from Sigma-Aldrich. PEO and HA were labeled with Coumarin 343 and DAPI, respectively, using a similar procedure through an EDC coupling reaction. For a typical labeling procedure: Polymer (PEO or $\mathrm{HA} ; 50 \mathrm{mg}$ ) was dissolved in water $(5 \mathrm{~mL})$ in a round-bottom flask (RBF). Subsequently, EDC (3.0 mg, $0.016 \mathrm{mmol})$ and DMAP (3.0 $\mathrm{mg}, 0.025 \mathrm{mmol}$ ) were added, and the resulting solution was stirred at room temperature for $20 \mathrm{~min}$. Finally, the aqueous solution of dye (Coumarin 343: $4.0 \mathrm{mg}$, $0.015 \mathrm{mmol}$; or DAPI: $5.0 \mathrm{mg}, 0.014 \mathrm{mmol}$ ) in $5 \mathrm{~mL}$ was added dropwise to the above RBF. The reaction mixture was stirred overnight at room temperature, then dialyzed using a cellulose dialysis tube (with $14 \mathrm{kDa}$ cutoff molecular weight) against distilled water (approx. $2 \mathrm{~L}$ ) overnight to remove the unreacted dye molecules.

\section{RESULTS AND DISCUSSION}

We studied particle migration in a microfluidic channel with the simultaneously applied pressure-driven flow and parallel DC electric field. We suspended $6 \mu \mathrm{m}$ diameter PS particles $\left(10^{3}\right.$ particles $\left./ \mathrm{mL}\right)$ in three different solutions: DI water, 500 ppm 5 MDa PEO-based viscoelastic solution, and $1000 \mathrm{ppm}$ 1.5 MDa HA-based viscoelastic solution. Solutions were pumped through the PDMS microchannel while DC electric field was applied simultaneously. The electric field generates electrophoretic motion due to the nonzero zeta potential of the suspended particles in each solution.

Initially, particle migration was studied for a pressure-driven flow without applying DC electric field (Figure 2d). Then, the electric field was simultaneously applied with the pressuredriven flow; two different tests were performed by changing the direction of the electrophoretic motion of the PS particles: concurrent where the pressure-driven flow is in the same direction as the electrophoretic force and countercurrent where the pressure-driven flow is in the opposite direction to the electrophoretic force. Shear dependent viscosities of the sample solutions were measured using a rotary rheometer (Supporting Information, Figure S-1). Volumetric flow rate $(Q)$, Reynolds number ( $\mathrm{Re})$, and Weissenberg number (Wi) were calculated as given in Supporting Information, Note S-6. Relaxation time $(\lambda)$ for dilute viscoelastic solutions was calculated using the Zimm relaxation theory (Supporting Information, Note S-2).

Our experimental outputs are summarized in Figure $2 \mathrm{~d}$ to display the particle equilibrium positions at the cross section of the observation window. Under the pressure-only case, particles in DI water show no cross-stream migration. However, particles in PEO- and HA-based viscoelastic solutions migrate to the channel center due to the elastic lift force, which pushes the particles to low shear rate regions. ${ }^{28-30}$ In the concurrent test, particles in DI water and HA-based viscoelastic solution migrate toward the walls. However, 


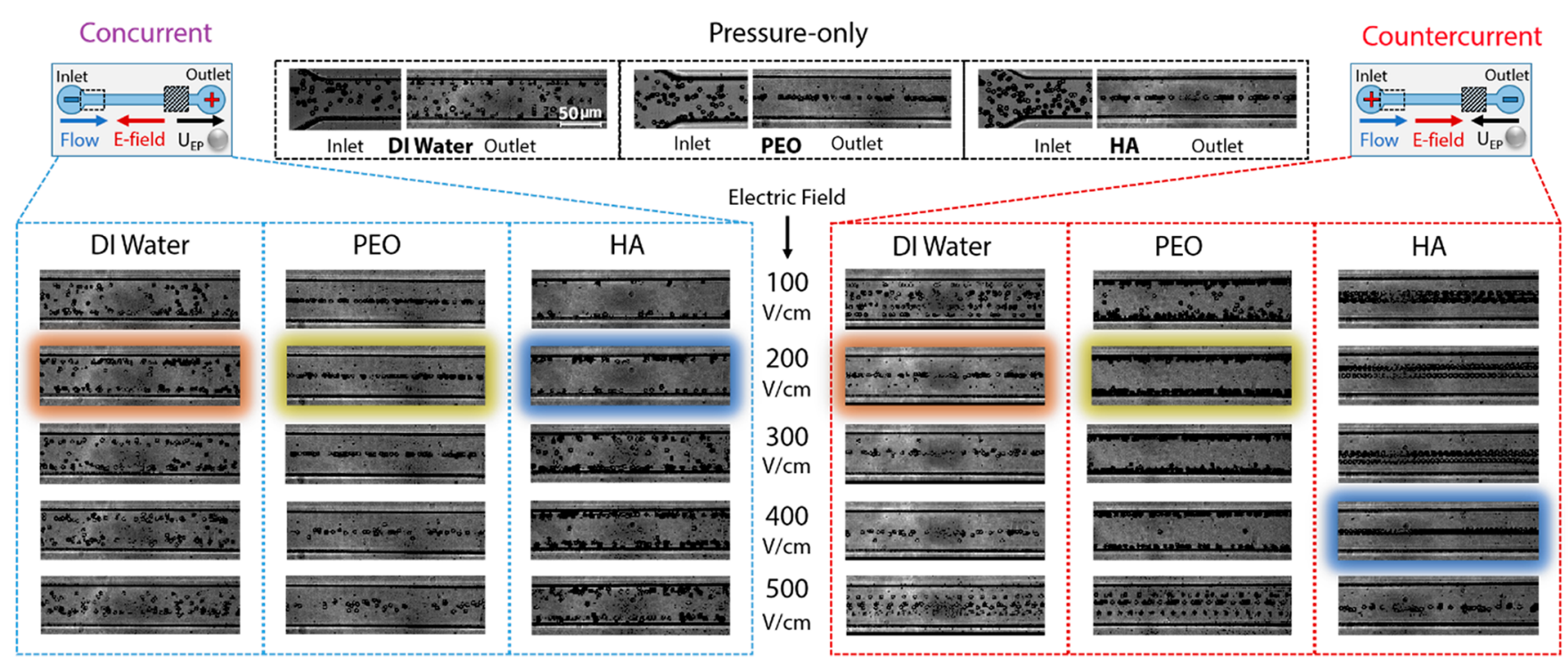

Figure 3. Top-view image-stacked photos of high-speed camera recordings for particle migration under pressure-driven flow and simultaneously applied pressure-driven flow and DC electric field. Three particle suspensions were prepared in DI water, PEO-, and HA-based viscoelastic solutions. Particle migration was observed at the outlet during concurrent and countercurrent tests. The electric field magnitude was varied between 100 and $500 \mathrm{~V} / \mathrm{cm}$. The best focusing performances were highlighted for each case. In the concurrent experiment, suspended particles migrated to the channel walls for DI water and HA, and the channel center for the PEO solution. Particles were oppositely aligned in countercurrent experiments.

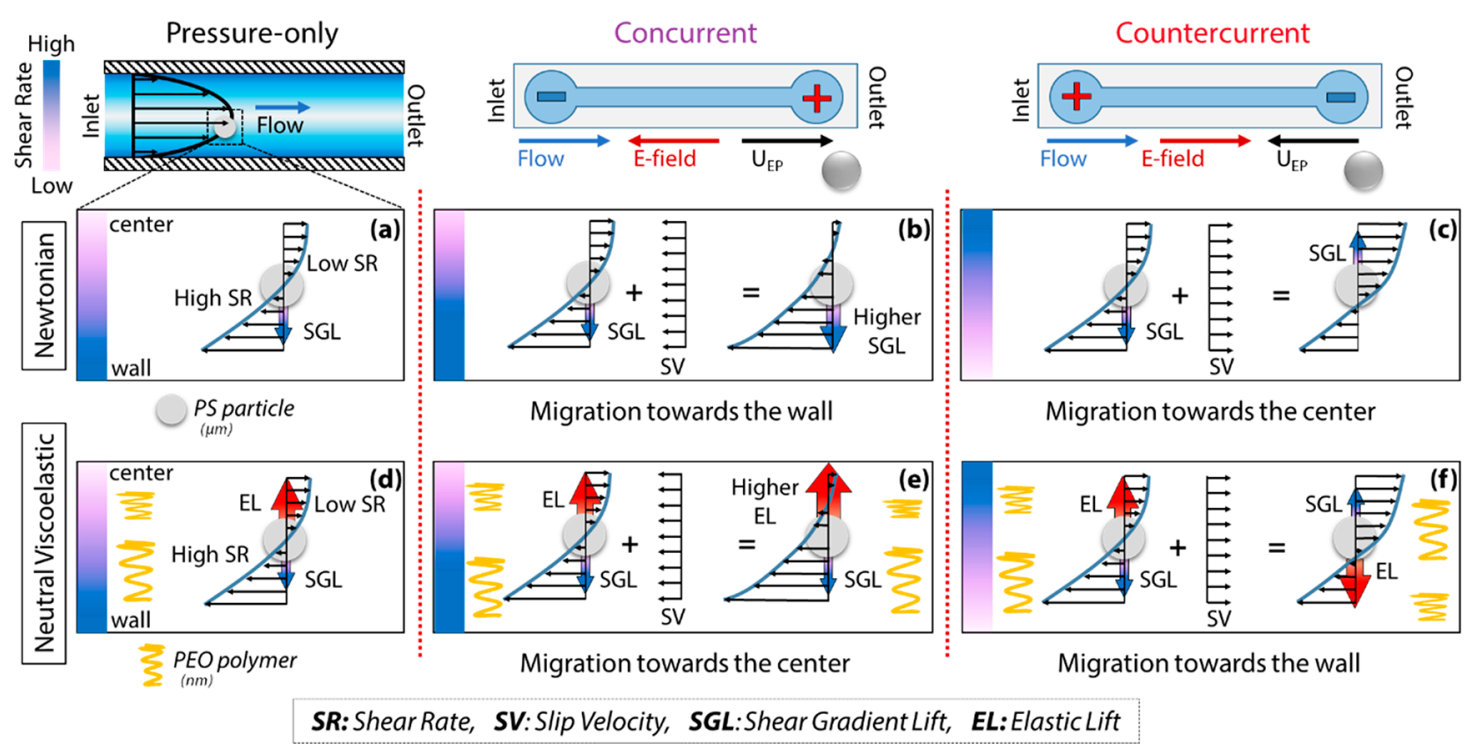

Figure 4. Illustrative explanation of particle migration in Newtonian and neutral viscoelastic solutions. Poiseuille flow generated in the microchannel with a pressure-driven flow. The particle is considered to be stationary relative to the fluid. Schematic explanation of particle migration in the Newtonian solution for (a) pressure-only, (b) concurrent, and (c) countercurrent cases. Schematic explanation of particle migration in the neutral viscoelastic solution for (d) pressure-only, (e) concurrent, and (f) countercurrent cases. Green spring, blue, and red arrows represent the PEO polymer stretching, shear gradient lift force, and elastic lift force, respectively. The color-chart on the left-hand side of the channel represents the shear gradient profile.

particles in the PEO-based viscoelastic solution migrate toward the channel center.

In the countercurrent test, the migration results are the opposite. It was reported earlier that particle migration in DI water and viscoelastic solutions are opposite to each other under the same experimental conditions. ${ }^{21}$ However, our results with HA-based viscoelastic solutions are controversial. Equilibrium positions of particles in HA-based viscoelastic solution are similar to DI water. We are hypothesizing that the polyelectrolyte nature of $\mathrm{HA}$ is the main reason for the difference observed with PEO- and HA-based viscoelastic solutions in EVM. Experimental evidence for our hypothesis is presented in the following sections.

Particle Migration under Simultaneously Applied Pressure-Driven Flow and DC Electric Field. Figure 3 shows the image stacked photos from the top-view video recordings at the channel outlet for simultaneously applied pressure-driven flow and DC electric field. The single stream of particles in the middle of the channel indicates migration to the channel center, while the two streams at the channel edge 


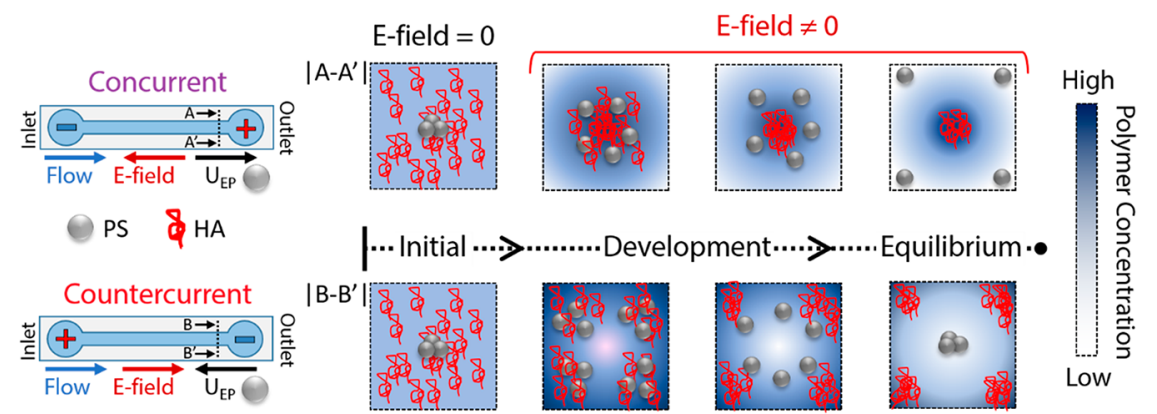

Figure 5. Development of particle migration in HA-based viscoelastic solutions at the outlet cross section of the channel under the simultaneously applied pressure-driven flow and electric field. Particles are initially centrally focused under pressure-only flow without the electric field. After an electric field is applied, gradual particle and polyelectrolyte migration are shown. In the concurrent case, HA polymers start to migrate toward the center. Resultantly, HA polymer concentration is increased at the channel center, and a concentration gradient is developed at the channel cross section (more viscoelastic at the center, more Newtonian at the walls). Increase in polymer concentration at the channel center generates a shearthinning profile. Particles migrate toward the walls over time. A similar mechanism applies in countercurrent case in the opposite direction. The blue color gradient bar represents the concentration gradient at the channel cross section. The color chart on the right represents the polymer concentration gradient.

indicate migration to the channel corners as schematically shown in Figure 2d. Pressure difference $(P)$ is kept constant at $30 \mathrm{mbar}$ for DI water ( $Q: 58 \mu \mathrm{L} / \mathrm{h}$, Re: 0.33 , Wi: 0$), 50 \mathrm{mbar}$ for PEO (Q: $56 \mu \mathrm{L} / \mathrm{h}, \mathrm{Re}: 0.18, \mathrm{Wi}: 4.39, \mathrm{El}: 23.6$ ), and 50 mbar for HA (Q: $2 \mu \mathrm{L} / \mathrm{h}, \mathrm{Re}: 0.0004, \mathrm{Wi}: 0.07, \mathrm{El}: 202$ ) solutions. The results of the concurrent and countercurrent experiments are given in Figure 3 for increasing the electric field magnitude $(0-500 \mathrm{~V} / \mathrm{cm})$.

The electrophoretic mobility is determined experimentally by tracking the motion of particles under the electric field without applying pressure-driven flow. ${ }^{31}$ The magnitude of electrophoretic velocity $\left(U_{\mathrm{EP}}\right)$ and electrophoretic mobility $\left(\mu_{\mathrm{EP}}\right)$ for both the concurrent and countercurrent modes are 0.4 and $0.15 \mathrm{~mm} / \mathrm{s} ; 2.0 \times 10^{-8} \mathrm{~m}^{2} /(\mathrm{V} \cdot \mathrm{s})$ and $0.75 \times 10^{-8} \mathrm{~m}^{2} /$ (V.s) for PEO and HA solutions, respectively, at $200 \mathrm{~V} / \mathrm{cm}$.

Pressure, flow rate, $\mathrm{Re}$, and $\mathrm{Wi}$ are kept constant during these experiments. Pressure-only experiments are performed to find the optimal pressure for central viscoelastic focusing for both PEO and HA viscoelastic solutions (Supporting Information, Figure S-2). At 50 mbar, a well-aligned single train of particles is achieved at the center of the channel, and the viscoelastic focusing is achieved for both solutions. In all cases, the particles are arbitrarily distributed at the channel inlet (Supporting Information, Figure S-3).

The best focusing results are highlighted in Figure 3. At 200 $\mathrm{V} / \mathrm{cm}$ concurrent experiments, particles are focused at the channel wall for DI solution, whereas they are focused at the center for the PEO solution. More interestingly, the second viscoelastic solution, HA, yielded the same results observed in the DI water medium. For each medium, the equilibrium particle positions are reversed for countercurrent experiment.

Although the two viscoelastic solutions (PEO and $\mathrm{HA}$ ) show very similar viscoelastic focusing behavior in the pressure-only regime $(0 \mathrm{~V} / \mathrm{cm})$, they give opposite migration results when the electric field was applied together with the pressure-driven flow. To our best knowledge, such an observation has not been reported so far. A comprehensive analysis of the governing forces is required to explain these intriguing results and the underlying mechanism of EVM.

Principle of EVM Using Dominant Lift Forces. It should be noted that PEO is a neutral polymer, ${ }^{32}$ and $\mathrm{HA}$ is a polyelectrolyte due to $\mathrm{Na}^{+}$backbones in the polymeric chain. ${ }^{27}$ Therefore, we divide the explanation for viscoelastic solutions into two parts. In Part-1, we explain the difference between the Newtonian and neutral viscoelastic solution (Figure 4). In Part-2, we focus on the polyelectrolyte viscoelastic solution and its similarity with Newtonian results (Figure 5). Figure 4 schematically explains the particle migration in Newtonian and neutral viscoelastic solutions under simultaneously applied pressure-driven flow and electric field.

Part 1a: Particle Migration inside Newtonian Medium. Pressure driven flow in a microchannel generates a quadratic velocity profile. Suspended particle in laminar flow is under the influence of this velocity profile. If an arbitrarily positioned particle is considered to be stationary relative to the fluid velocity, it experiences different velocity magnitudes due to the shear gradient in the channel (Figure 4a). ${ }^{33}$ The velocity difference around the particle generates a shear gradient lift (SGL) force, which pushes the particle to low velocity, high shear rate regions, channel walls. Particles are always under the effect of SGL; yet, to generate considerable migration with SGL force, high Re is required. ${ }^{34}$

The use of the DC electric field, in addition to the pressuredriven flow, changes the velocity of the particle relative to the fluid velocity due to the electrophoretic force acting on the particle. The additional DC electric field generates a slip velocity depending on the charge of the particle. ${ }^{21,35}$ The electro-osmotic flow is neglected in comparison to the pressure-driven flow. In the concurrent experiment, the velocity of the particle increases relative to the fluid velocity due to the electrophoretic force. This increase is shown in Figure $4 \mathrm{~b}$ as a slip velocity. When the slip velocity is superposed with the fluid velocity, the relative velocity difference on both sides of the particle becomes higher compared to the pressure-only case. Higher velocity difference generates higher SGL force, which pushes the particle toward the channel walls (Figure $4 \mathrm{~b}$ ). In the countercurrent experiment, the electric field, electrophoretic force, and slip velocity directions are reversed. After superposing the slip velocity and particle velocity streamlines, high and low shear rate regions flipped at the affinity of the particle; thus, the direction of SGL also flipped, and particle migrates toward the channel center (Figure 4c).

Part 1b: Particle Migration inside Viscoelastic Medium. In a pressure-driven flow, viscoelastic polymers are stretched along with the applied shear. The shear gradient in 


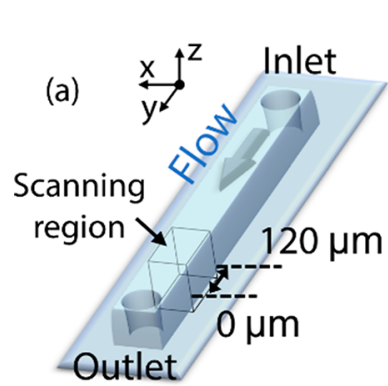

(b)

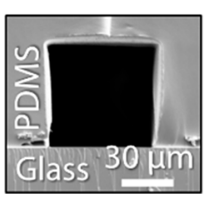

Pressure-only

Concurrent

Countercurrent

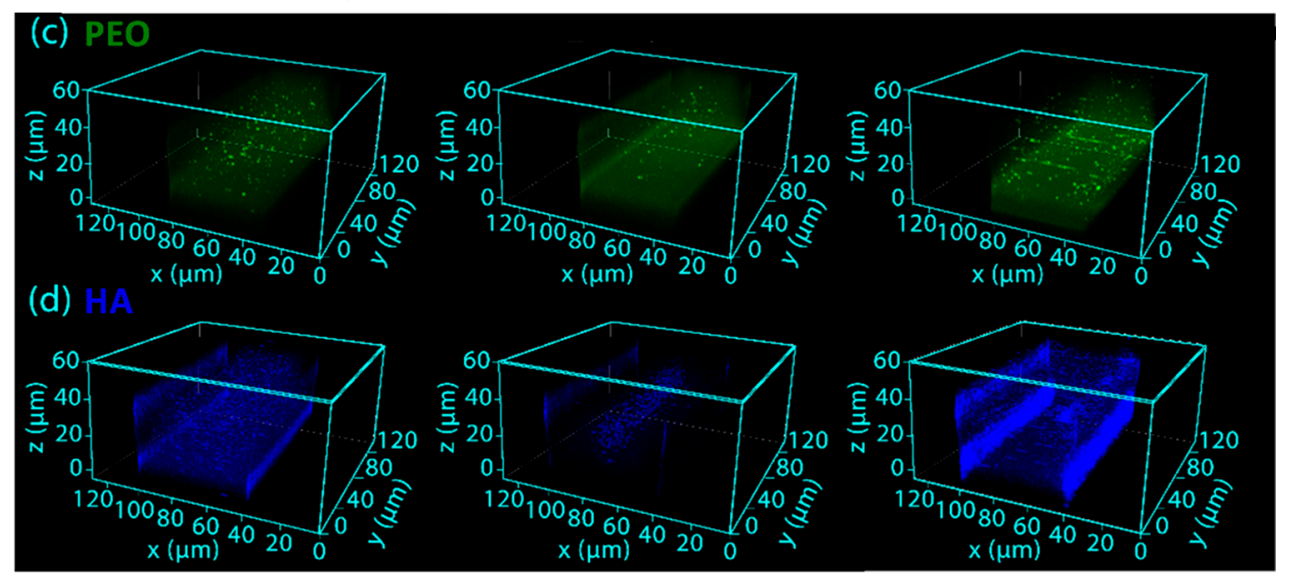

Figure 6. Confocal imaging results for the distribution of fluorescently tagged polymers at the channel cross section near the outlet. Polymers are observed in three different modes: pressure-only, concurrent, and countercurrent. Applied electric field is $200 \mathrm{~V} / \mathrm{cm}$ for the concurrent and countercurrent modes. (a) Schematic drawing of the channel and scanning volume. The confocal scanning region is $60 \times 120 \times 120 \mu \mathrm{m}^{3}$. The microfluidic channel was placed onto the motorized sample slider. (b) Scanning electron microscope (SEM) image of the channel cross section (60 $\mu \mathrm{m} \times 50 \mu \mathrm{m}$ ) showing the PDMS side-walls and channel top and the glass channel bottom. (c) Coumarin 343 tagged-PEO. Uniform polymer distribution is observed in all modes due to the electrical neutrality of PEO. (d) DAPI tagged-HA. Polymer concentration distribution varies depending on the presence and polarity of the electric field due to the polyelectrolyte nature of HA. Note that we applied gamma correction with maximum intensity threshold on the fluorescent images; see Supporting Information, Figure S-5 for more information.

the channel causes nonuniform stretching of polymers, which increases at a high shear rate as schematically shown in Figure 4d. Nonuniform polymer stretching generates an elastic lift (EL) force on the suspended particles directing from high shear rate to low shear rate regions, from channel walls to center. ${ }^{29}$ At low Re, SGL is negligible compared to EL. Thus, the particle is attracted to the channel center under the pressure-only condition (Figure $4 \mathrm{~d}$ ). In the concurrent case, the slip velocity enhances the shear stress around the particle and generates higher EL force directing toward the channel center in the concurrent experiment (Figure 4e). ${ }^{24}$ In the countercurrent case, the high and low shear rate regions around the particle are reversed, which flips the polymer stretching region in the affinity of the particle. Consequently, the EL force direction is flipped, directing the particles toward the channel walls (Figure 4f).

Part 2: Nonuniform Viscoelasticity. Under the pressureonly case, viscoelastic polymers are stretched due to local shear rate and are migrated away from the walls of the channel; yet, this migration is only observed at the proximity of the channel walls. ${ }^{17}$ Neutral polymers are not affected (not stretched or migrated) by the electric field under the simultaneous use of pressure-driven flow and DC electric field. However, the crossstream migration is observed for the polyelectrolytes such as $\lambda$ DNA (and HA in our experiment) due to the electrophoretic force-induced electro-hydrodynamic interactions (EHI). ${ }^{15,17}$

We hypothesize that viscoelastic polymer concentration at the channel cross section stays uniform for neutral polymers. However, for the polyelectrolytes, the polymer concentration locally changes, which in turn generates a nonuniform viscoelastic solution distribution inside the microchannel.

Figure 5 schematically illustrates the development of particle migration and nonuniform viscoelasticity for the HA-based viscoelastic solution for concurrent and countercurrent experiments. Initially, particles are centrally focused under the pressure-only regime where there is no electric field. In the concurrent case, HA molecules (under the influence of EHI) are progressively attracted to the channel center. Consequently, HA polymer concentration gradually increases around the center. This starts to generate a polymer concentration gradient at the channel cross section, resulting in a more Newtonian solution at the channel walls. At the channel center, the increase in HA concentration leads to a high-shear thinning behavior. In high shear-thinning viscoelastic solutions, particles migrate toward the channel walls under pressure-driven flow. ${ }^{13}$ High shear thinning behavior starts to play the dominant role for the particle migration so that particles are progressively pushed away from the center. At the steady-state, particles migrate to the more Newtonian regions (walls). In the countercurrent case, the focusing trend is entirely reversed. HA polymers start to migrate toward the walls. Then, the highshear thinning behavior is developed at the corners due to the HA concentration increase. Thus, the channel center becomes more Newtonian. At the steady-state, particles are focused to the channel center. This explanation clarifies the similarity of the focusing behavior in HA and Newtonian solutions, as experimentally shown in Figure 3.

Confocal Imaging of the Fluorescent Dye-Labeled Polymer Viscoelastic Solutions. To experimentally confirm our hypothesis, we labeled PEO and HA with fluorescent dyes Coumarin 343 and DAPI, respectively. The fluorescently labeled PEO and HA viscoelastic solutions were used to perform confocal imaging on polymer distribution along the flow axis (Figure 6a). Confocal microscope images are given in Figure $6 \mathrm{c}, \mathrm{d}$. We improved the contrast by gamma correction and maximum intensity threshold on the fluorescent images to clarify the concentration gradient. Raw confocal images, maximum intensity projected images, and fluorescent intensity profile across the channel cross section is given in Supporting Information, Figure S-5.

Coumarin tagged-PEO polymer concentration is uniform at the channel cross section near the outlet regardless of the presence or the direction of the electric field in Figure $6 c$, due to the electrically neutral nature of PEO. Figure $6 \mathrm{~d}$ 


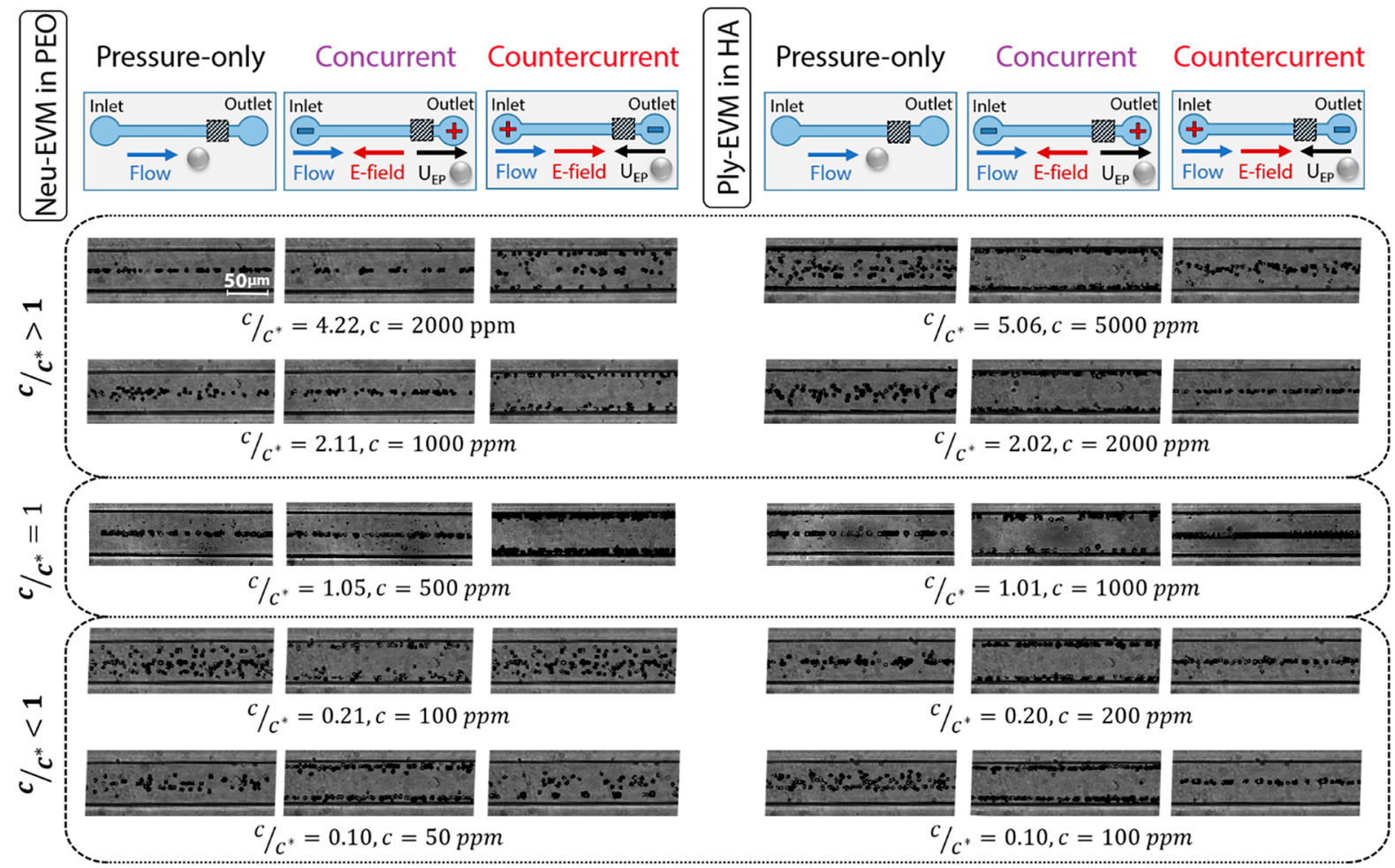

Figure 7. Top-view image-stacked photos of high-speed camera recordings to show the effect of polymeric concentration on particle equilibrium positions at the channel outlet for Neu-EVM and Ply-EVM. Particle migration at different polymeric concentration ratios, $c / c^{*}$, is studied for NeuEVM in PEO and Ply-EVM in HA solutions for pressure-only, concurrent, and countercurrent tests. The electric field is applied at $200 \mathrm{~V} / \mathrm{cm}$. Particle equilibrium positions in Neu-EVM shows a concentration-dependent transition when $c / c^{*}<1$. In Ply-EVM equilibrium, particle positions are the same at each concentration ratio. $c^{*}{ }_{\mathrm{PEO}}=473 \mathrm{ppm}$ and $c^{*}{ }_{\mathrm{HA}}=988 \mathrm{ppm}$.

demonstrates migration behavior for DAPI-tagged HA solution at the channel cross section near the outlet. In contrast to the PEO case, HA molecules respond to the changes in the electric field. When no electric field is applied (pressure-only mode), the polymer distribution is uniform at the cross section. In the concurrent mode, HA polymers migrate from walls to the channel center, showing higher fluorescence intensity at the center. In the countercurrent mode, polymers are highly populated near the channel corners (walls). Nonuniform polymer distributions in different modes at the channel cross section are due to the polyelectrolyte nature of HA molecules. The confocal observations on the behavior of molecules are in parallel with the experimental results in Figure 3 and our hypothesis schematically shown in Figure 5.

EVM for Varying Viscoelastic Polymer Concentrations. The experimental observations and EVM explained in the previous sections are performed at a specific polymer concentration ratio $c / c^{*}=1$, where $c$ is the polymeric concentration in solution and $c^{*}$ is the overlap concentration. This ratio defines the dilute to semidilute crossover regime of polymer solutions. In dilute solutions, $c$ is substantially lower than $c^{*}$, and the viscoelastic solution shows no effective entanglement with ideal viscous flow behavior. The viscoelastic solution is considered to be semidilute if $c \geq c^{*}$, where polymer coils start to overlap and entanglement fluctuations occur. ${ }^{36,37}$

Elastic lift force, which is a function of polymer concentration, plays a key role in viscoelastic particle focusing. Hence, it is critical to investigate the viscoelastic polymer concentration dependence of EVM. To support the underlying mechanism for neutral PEO and polyelectrolyte HA viscoelastic solutions, we define two types of EVM based on the charge of the viscoelastic solution: neutral-EVM (NeuEVM) and polyelectrolyte-EVM (Ply-EVM).

We demonstrate the effect of polymeric concentration on the equilibrium particle positions at the channel outlet under simultaneously applied pressure-driven flow and DC electric field in Figure 7. Three regimes, $c / c^{*}>1 ; c / c^{*}=1 ; c / c^{*}<1$, are considered by changing the polymer concentration of PEO and $\mathrm{HA}$ viscoelastic solutions.

Neu-EVM in PEO experiments yielded an interesting transition depending on the polymer concentration ratio. When $c / c^{*}<1$, the equilibrium particle positions were reversed, which are similar to the Newtonian solution (as shown in Figure 3). However, Ply-EVM in HA experiments showed that particle equilibrium positions did not change at varying polymer concentration ratios.

These experiments show the major difference between the mechanisms of Neu-EVM and Ply-EVM. In Neu-EVM, the migration profile is dictated by the forces effective on the suspended particles. The uniform neutral polymer concentration profile is preserved throughout the microchannel. Hence, lowering the polymeric concentration below $c^{*}$, namely, $c / c^{*}<1$, dramatically decreases the elastic lift force leading to opposite migration results in Figure 7.

On the other hand, Ply-EVM is based on polyelectrolyte migration creating a Newtonian medium profile in the channel cross section. This profile is equivalent to coflow of Newtonian and viscoelastic mediums. In the equilibrium state, suspended particles migrate to Newtonian regions. Thus, the Ply-EVM results shown in Figure 7 are independent of polymer concentration for the range studied in this work. 


\section{CONCLUSION}

This study demonstrates bidirectional particle migration under simultaneously applied pressure-driven flow and electric field in Newtonian, PEO neutral viscoelastic and HA polyelectrolyte viscoelastic solutions. When the direction of pressure flow and electrophoretic force are concurrent, particles migrate toward the channel walls in Newtonian and HA solutions and toward the channel center in PEO solution. Surprisingly, particles in the HA polyelectrolyte viscoelastic solution exhibit a similar migration profile to the ones in the Newtonian solution. Even though HA and PEO polymers have similar viscoelastic properties, they show an opposite migration profile as a response to applied electric fields. To explain such behavior, we introduce EVM theory, which takes viscoelastic polymer charges into account.

Confocal imaging of fluorescent stained PEO- and HA-based viscoelastic solutions demonstrates that in neutral viscoelastic solutions (Neu-EVM), electric field does not induce polymer migration and only modulates the elastic lift force. In contrast, in polyelectrolyte viscoelastic solutions (Ply-EVM), electric field induces migration of polyelectrolytes, the direction of which depends on the polarity of the electric field. As a result of polyelectrolyte migration, a cross-sectional polymer concentration gradient is generated at the microchannel.

To further investigate the difference between Neu-EVM and Ply-EVM, we performed migration experiments at varying polymeric concentrations. Particle equilibrium positions show concentration-dependent results for Neu-EVM when $c / c^{*}<1$. However, in Ply-EVM, particle equilibrium positions are similar to the results in a Newtonian solution, and a polymer concentration-independent migration profile is obtained.

These results lead to a unifying EVM theory, explaining the particle migration results in simultaneously applied pressuredriven flow and electric field. We present the fundamental understanding and explain the particle migration behavior in most commonly used carrier fluids, Newtonian, neutral viscoelastic, and polyelectrolyte viscoelastic solutions.

Implementation of EVM theory in microfluidics presents an opportunity for various fields: (i) EVM might provide a good environment to study the elastic instabilities in complex fluids for different viscoelastic solutions at varying $\mathrm{Re}$ and $\mathrm{Wi}$ numbers. $^{38,39}$ (ii) Dilute to semidilute crossover regime, $c^{*}$, of polymer solutions can be detected in Neu-EVM by monitoring the particle equilibrium positions. (iii) EVM enhances the central viscoelastic focusing at smaller distance in microchannel (Supporting Information, Note S-7 and Figure S-4). (iv) Ply-EVM gives a focusing profile independent of polymer concentration. Its implementation in microfluidic flow cytometry can provide $3 \mathrm{D}$ particle focusing for the miniaturization of flow cells. ${ }^{1,3}$ (v) Cells, polyelectrolytes, DNA, and proteins in a complex medium such as whole blood or biological serums can be precisely focused and separated according to their charge and polymeric size for electrophoresis or chromatography applications. ${ }^{25}$

\section{ASSOCIATED CONTENT}

\section{s) Supporting Information}

The Supporting Information is available free of charge at https://pubs.acs.org/doi/10.1021/acs.analchem.9b05620.

Movie S-1: Cross-sectional imaging near the outlet for HA-DAPI (MOV)
Movie S-2: Cross-sectional imaging near the outlet for PEO-Coumarin (MOV)

Rheometer measurements for prepared solutions (Note S-1 and Figure S-1); estimation of effective relaxation time (Note S-2 and Tables S-1, S-2, and S-3); viscoelastic flow-focusing under only pressure-driven flow condition without electric field (Note S-4 and Figure S-2); Initial PS particle distribution at the channel inlet (Note S-5 and Figure S-3); Calculation of dimensionless numbers (Note S-6 and Tables S-4, S-5, S-6, and S-7); observation of enhanced viscoelastic focusing under Neu-EVM conditions (Note S-7 and Figure S-4); 2D maximum intensity projection images and intensity plots (Note S-8 and Figure S-5) (PDF)

\section{AUTHOR INFORMATION}

\section{Corresponding Author}

Caglar Elbuken - UNAM - National Nanotechnology Research Center, Institute of Materials Science and Nanotechnology, Bilkent University, 06800 Ankara, Turkey; 이이.org/00000001-8359-6871; Email: elbuken@unam.bilkent.edu.tr

\section{Authors}

Murat Serhatlioglu - UNAM - National Nanotechnology Research Center, Institute of Materials Science and Nanotechnology, Bilkent University, 06800 Ankara, Turkey

Ziya Isiksacan - UNAM - National Nanotechnology Research Center, Institute of Materials Science and Nanotechnology, Bilkent University, 06800 Ankara, Turkey

Melis Özkan - UNAM - National Nanotechnology Research Center, Institute of Materials Science and Nanotechnology, Bilkent University, 06800 Ankara, Turkey

Dönüs Tuncel - UNAM - National Nanotechnology Research Center, Institute of Materials Science and Nanotechnology, Bilkent University, 06800 Ankara, Turkey; @ orcid.org/00000001-7762-9200

Complete contact information is available at:

https://pubs.acs.org/10.1021/acs.analchem.9b05620

\section{Author Contributions}

M.S. and C.E. conceived the project. C.E. supervised the project. M.S. and C.E. designed the experiments. M.S. performed chip fabrication. M.S. and Z.I. performed the experiments. M.S. prepared figures and drafted the manuscript. Z.I. contributed to manuscript preparation and discussion. M.O. and D.T. performed fluorescent labeling and contributed to confocal experiments. All authors edited and revised the manuscript.

\section{Notes}

The authors declare no competing financial interest.

\section{ACKNOWLEDGMENTS}

The authors acknowledge support from Prof. Halil Ibrahim Unal and Dr. Omer Yunus Gumus during the characterization of viscoelastic polymers. The authors thank Prof. Carolyn Ren for her valuable discussions. The authors thank Talha Masood Khan for proof-reading the manuscript. The authors thank Mohammad Asghari for providing the fluorescent dyes. The authors thank Dr. Gokce Celik and Esra A. Karaaslan for their valuable contribution in confocal imaging experiments. Ziya Isiksacan is supported by ASELSAN Graduate Scholarship for Turkish Academics. Dr. Elbuken acknowledges the support 
from The Science Academy, Turkey, through the Young Scientist Award Program.

\section{REFERENCES}

(1) Serhatlioglu, M.; Asghari, M.; Tahsin Guler, M.; Elbuken, C. Electrophoresis 2019, 40 (6), 906-913.

(2) Asghari, M.; Serhatlioglu, M.; Ortaç, B.; Solmaz, M. E.; Elbuken, C. Sci. Rep. 2017, 7 (1), 12342-14.

(3) Etcheverry, S.; Faridi, A.; Ramachandraiah, H.; Kumar, T.; Margulis, W.; Laurell, F.; Russom, A. Sci. Rep. 2017, 7 (1), 1-8.

(4) Lim, E. J.; Ober, T. J.; Edd, J. F.; Desai, S. P.; Neal, D.; Bong, K. W.; Doyle, P. S.; McKinley, G. H.; Toner, M. Nat. Commun. 2014, 5, 4120

(5) Kang, K.; Lee, S. S.; Hyun, K.; Lee, S. J.; Kim, J. M. Nat. Commun. 2013, 4, 2567.

(6) Young Kim, J.; Won Ahn, S.; Sik Lee, S.; Min Kim, J. Lab Chip 2012, 12 (16), 2807-2814.

(7) Holzner, G.; Stavrakis, S.; Demello, A. Anal. Chem. 2017, 89 (21), 11653-11663.

(8) Asghari, M.; Cao, X.; Mateescu, B.; Van Leeuwen, D.; Aslan, M. K.; Stavrakis, S.; Demello, A. J. ACS Nano 2020, 14 (1), 422-433.

(9) Nam, J.; Namgung, B.; Lim, C. T.; Bae, J. E.; Leo, H. L.; Cho, K. S.; Kim, S. J. Chromatogr. A 2015, 1406, 244-250.

(10) Lu, X.; Xuan, X. Anal. Chem. 2015, 87 (22), 11523-11530.

(11) Liu, C.; Xue, C.; Chen, X.; Shan, L.; Tian, Y.; Hu, G. Anal. Chem. 2015, 87 (12), 6041-6048.

(12) Faridi, M. A.; Ramachandraiah, H.; Banerjee, I.; Ardabili, S.; Zelenin, S.; Russom, A. J. Nanobiotechnol. 2017, 15 (1), 1-9.

(13) Del Giudice, F.; Sathish, S.; D’Avino, G.; Shen, A. Q. Anal. Chem. 2017, 89 (24), 13146-13159.

(14) Zheng, J.; Yeung, E. S. Anal. Chem. 2002, 74 (17), 4536-4547.

(15) Arca, M.; Butler, J. E.; Ladd, A. J. C. Soft Matter 2015, 11 (22), 4375-4382

(16) Arca, M.; Ladd, A. J. C.; Butler, J. E. Soft Matter 2016, 12 (33), 6975-6984.

(17) Kekre, R.; Butler, J. E.; Ladd, A. J. C. Phys. Rev. E 2010, 82 (5), $1-4$.

(18) Montes, R. J.; Butler, J. E.; Ladd, A. J. C. Electrophoresis 2019, 40 (3), 437-446.

(19) Kim, Y. W.; Yoo, J. Y. Lab Chip 2009, 9 (8), 1043-1045.

(20) Kim, Y. W.; Yoo, J. Y. J. Micromech. Microeng. 2015, 25 (2), 027002-027006.

(21) Li, D.; Xuan, X. Phys. Rev. Fluids 2018, 3 (7), 1-13.

(22) Cevheri, N.; Yoda, M. Langmuir 2014, 30 (46), 13771-13780.

(23) Cevheri, N.; Yoda, M. Lab Chip 2014, 14 (8), 1391-1394.

(24) Ranchon, H.; Malbec, R.; Picot, V.; Boutonnet, A.; Terrapanich, P.; Joseph, P.; Leïchlé, T.; Bancaud, A. Lab Chip 2016, 16 (7), 1243-1253.

(25) Andriamanampisoa, C. L.; Bancaud, A.; Boutonnet-Rodat, A.; Didelot, A.; Fabre, J.; Fina, F.; Garlan, F.; Garrigou, S.; Gaudy, C.; Ginot, F.; et al. Anal. Chem. 2018, 90 (6), 3766-3774.

(26) Choudhary, A.; Li, D.; Renganathan, T.; Xuan, X.; Pushpavanam, S. arXiv Prepr. 2019, 1, na.

(27) Dubrovskii, S. A.; Zelenetskii, A. N.; Uspenskii, S. A.; Khabarov, V. N. Polym. Sci., Ser. A 2014, 56 (2), 205-210.

(28) Del Giudice, F.; Romeo, G.; D’Avino, G.; Greco, F.; Netti, P. A.; Maffettone, P. L. Lab Chip 2013, 13 (21), 4263.

(29) D’Avino, G.; Romeo, G.; Villone, M. M.; Greco, F.; Netti, P. A.; Maffettone, P. L. Lab Chip 2012, 12 (9), 1638-1645.

(30) Yang, S.; Lee, S. S.; Ahn, S. W.; Kang, K.; Shim, W.; Lee, G.; Hyun, K.; Kim, J. M. Soft Matter 2012, 8 (18), 5011.

(31) Liu, Z.; Li, D.; Song, Y.; Pan, X.; Li, D.; Xuan, X. Phys. Fluids 2017, 29 (10), 102001.

(32) Shirahama, K.; Himuro, A.; Takisawa, N. Colloid Polym. Sci. 1987, 265 (2), 96-100.

(33) Martel, J. M.; Toner, M. Annu. Rev. Biomed. Eng. 2014, 16 (1), 371-396.

(34) Amini, H.; Lee, W.; Di Carlo, D. Lab Chip 2014, 14, 27392761.
(35) Saffman, P. G. J. Fluid Mech. 1965, 22, 385-400.

(36) Litmanovich, E. A.; Syaduk, G. V.; Lysenko, E. A.; Zezin, A. B.; Kabanov, A. V.; Kabanov, V. A. Polym. Sci., Ser. A 2006, 48 (9), $997-$ 1003.

(37) Mezger, T. G. The Rheology Handbook For Users of Rotational and Oscillatory Rheometers, 4th ed.; Vincentz Network: Hanover, 2014.

(38) Galindo-Rosales, F. J.; Campo-Deaño, L.; Sousa, P. C.; Ribeiro, V. M.; Oliveira, M. S. N.; Alves, M. A.; Pinho, F. T. Exp. Therm. Fluid Sci. 2014, 59, 128-139.

(39) Li, D.; Xuan, X. Microfluid. Nanofluid. 2018, 22 (4), 22-49. 